\title{
THE RIDLEY LENS IMPLANT*
}

\author{
BY \\ EDWARD EPSTEIN \\ Johannesburg
}

THIS report is based on 84 cases. The youngest patient was 9 years old and the oldest 80 years at the time of surgery. The first operation was done in November 1951, and the last in December 1955.

\section{Material}

This series of cataracts includes eighteen traumatic, three complicating heterochromic cyclitis, one congenital, and the rest of indefinite aetiology-so-called senile cataracts (though several of these patients were only 40 years old)-and probably including cataracts due to contusions and other physical and toxic causes.

They were all unilateral except the one case of congenital cataract and two cases in the senile group. However, a further thirty cases in the senile group had very slight changes in the second eye at the time of operation, but still had a visual acuity of $6 / 6$ or better. Of these thirty cases, eight have shown a further deterioration in the unoperated eye, so that one has had a second implant after 2 years and four others will require the second eye to be done shortly. One case had had a left extraction in 1949, as the right eye had early changes which, it was thought, were likely to progress rapidly. However, when seen by me in 1952 the unoperated eye still had a visual acuity of 6/6 ptly. As an extracapsular round-pupil extraction had been done, a lens implant was possible. The visual acuity to-day, 4 years later, is right still $6 / 9+$, and left $6 / 5$.

\section{Results}

Of these 84 cases, twelve were failures for probable causes to be discussed below. This seems a high percentage, but includes six or seven cases of an experimental nature where the patient was warned a good result might not be obtained.

Of the remainder, thirteen cases obtained $6 / 5$ visual acuity with correction; $23-6 / 6 ; 23-6 / 9$; six-6/12; four-6/18; two-6/24; and one-6/60.

The last had extensive retinal degenerative changes.

The two with 6/24 had macular lesions: one, a "hole" of unknown aetiology, the other a lesion caused by an intra-ocular foreign body that had impinged on the macula before falling back into the vitreous.

The four with 6/18 included one case of heterochromic cyclitis in which the eye had been blind for nearly 40 yrs and after surgery showed a probable macular lesion and a rather hazy vitreous. The other eye, however, had

* Received for publication December 31, 1956. 
$6 / 9$ vision and the patient is delighted with the result. Two others with 6/18 were cases of traumatic cataract and included corneal damage so that a near central leucoma complicated the issue. The fourth turned out to have had unilateral high myopia.

The six cases achieving 6/12 vision included one of heterochromic cyclitis; this eye had. been blind for $10 \mathrm{yrs}$, and there was a fair degree of vitreous haze. The other five cases also showed various degrees of vitreous haze and posterior capsular opacities. It is expected that some of these will improve and in others it is anticipated that a posterior needling will have to be done.

\section{Technique}

The surgical technique has been slightly modified over the years. The following points indicate the present procedure. Premedication includes Diamox $500 \mathrm{mg}$. and chlorpromazine $50 \mathrm{mg}$. the night before and 2 hours pre-operatively. I prefer the eye to be as soft as possible. Topical Decicaine 1 per cent., homatropine 5 per cent., and cocaine 3 per cent., with retrobulbar procaine 4 per cent., or xylocaine 2 per cent. with epinephrine and hyalase are used for mydriasis and local anaesthesia. Lid akinesia is obtained by the usual methods.

A lateral canthotomy is done routinely. Apart from allowing a wider exposure of the eye, I believe it helps to inhibit any tendency to close the lids tightly in the post-operative period. A 3 to $4-\mathrm{mm}$. conjunctival flap is raised by scissors dissection before cutting the section. With the eye very soft, cutting a large $\left(180^{\circ}\right)$ von Graefe section without damaging the iris can be difficult. I therefore prefer to cut a smaller section and enlarge with scissors to the 3 to 9 o'clock diameter.

Two double-armed $6 \times 0$ silk sutures, with Grieshaber or Jameson Evans needles, are used to insert four edge-to-edge sutures so that when tied the result is two mattress-sutures on edge.

A peripheral iridotomy at 12 o'clock is done next. For the capsulectomy I prefer to use a cystitome to cut as large a flap of capsule as possible. It is then completed with forceps. I have adopted this procedure to remove as much of the anterior capsule as possible. I have not found forceps capsulectomy by itself to give an adequate removal of the anterior capsule, and occasionally, with a tough capsule, weak zonule, and blunt toothed-forceps, an intracapsular extraction is unintentionally done. For a similar reason I hesitate to pull on remaining firmly-attached tags of capsule.

When the acrylic implant is being inserted the corneal flap is held well up with fine non-toothed forceps, so that the endothelium is not rubbed as the lens is introduced (Fig. 1, overleaf). The lens, held by a pair of straight iris forceps, is pushed well under the iris towards 6 o'clock until the largest diameter is engaged in the pupil. It is then slightly withdrawn so that the optical axis is judged to be a little above the normal pupillary centre. In 


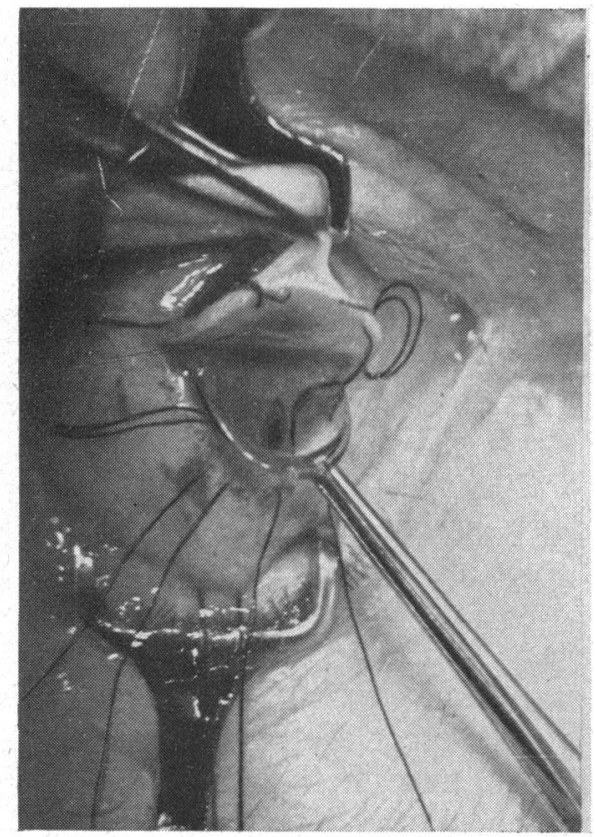

FIG. 1.-Surgeon's view, introducing lens. Note conjunctival-corneal flap held well up by non-toothed fine forceps, whilst acrylic is fixed by iris forceps. Magnified iridotomy is visible through lens, which in this case is the experimental larger type mentioned in the text.

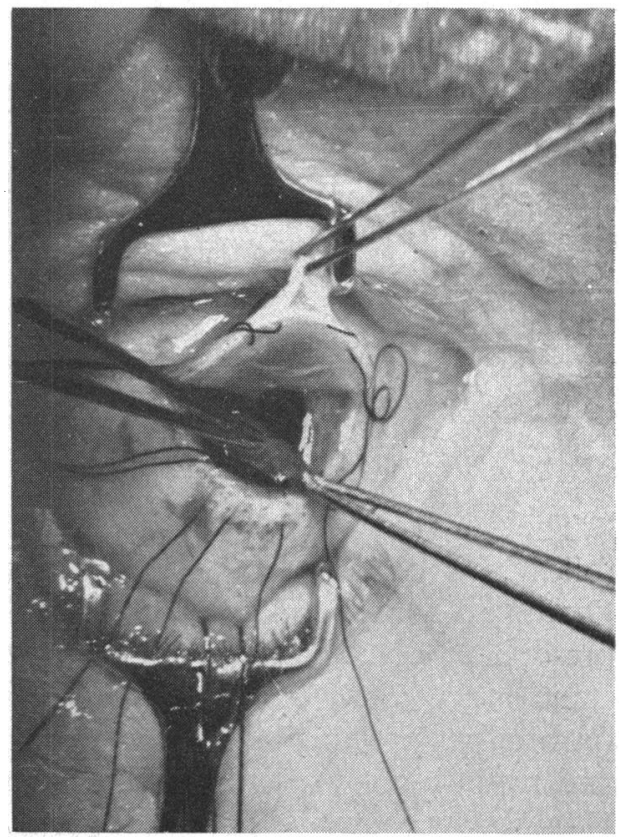

FIG. 2.-Iris in region of 12 o'clock being drawn round the lens. Note conjunctivalcorneal flap now fixed by assistant. Forceps that had been holding flap now used as a probe to steady lens.

this manoeuvre the iris moves up with the lens so that the largest diameter is not disengaged. An assistant then takes over the corneal flap whilst the non-toothed fine forceps that had been holding it are applied with the jaws closed to press as a probe near the centre of the lens to steady it (Fig. 2). The iris forceps that had been holding the lens itself are then released. The iris about the $12 \mathrm{o}$ 'clock meridian is picked up by these forceps and pulled around and over the lens, thus completely covering it. I prefer forceps to Ridley's iris spike to pull the upper part of the iris around the upper pole of the lens, for I have seen the spike perforate the iris and draw vitreous and this in the hands of a capable surgeon.

Drops of histamine with pilocarpine* are usually instilled into the anterior chamber at this point to get a good miosis if the pupil does not contract spontaneously. The non-toothed fine forceps that had been applied as a probe to steady the lens is used to depress the lens against the posterior capsule to allow the iris to contract well over the lens. The sutures are drawn to close the wound and are now completed by being passed through the conjunctival flap (Fig. 3, opposite). * Histamine 2\% with pilocarpine 5\%, available in sterile 1-ml. ampoules from Saphar Laboratories Pty. Ltd., 
Fig. 3.-The two mattress-on-edge sutures have been drawn and are being completed by passing the needles through the conjunctiva. A largish bubble has been introduced into the anterior chamber.

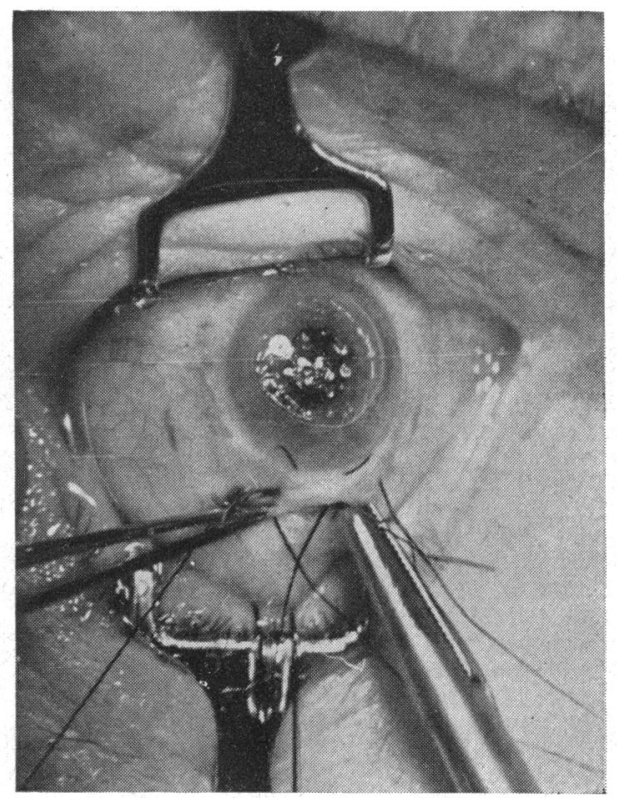

A bubble of air is injected as soon as possible into the anterior chamber to separate endothelium from the acrylic implant in the pupil area. The centration of the acrylic is inspected, and if unsatisfactory a small blunt lens hook or iris repositor with a suitable double curve is introduced to press gently on the lens through the pupil. Pressure with a second repositor just outside the limbus is then applied to move the lens into the correct position. Any form of manipulation that presses the cornea and therefore the endothelium directly against the lens or indirectly through the iris must be avoided. The manipulation just described avoids this and also allows the lens to move relative to the iris. Pressure at the limbus alone, especially without any bubble of air in the anterior chamber, may move the lens, but the iris may move with it and be put on the stretch, subsequently tending to move the lens back to an eccentric position.

In some eyes it is easy to observe the position of the lens as its outline is discernible through the iris. In other cases, especially those with tough brown irides, it is difficult to see the position of the lens. To help with centration I have tried placing a small drop of blood on to the centre of the anterior face of the lens which is left to dry. It helps a great deal in some cases, but in those requiring manipulation the small blood clot is unfortunately soon wiped off.

When centration is satisfactory and the sutures have been tied, a few minims of hydrocortisone $25 \mathrm{mg}$. $/ \mathrm{ml}$. are instilled into the anterior chamber, with enough air to get a good depth (Figs 4 and 5, overleaf). A further $\frac{1}{4} \frac{1}{2} \mathrm{ml}$. is injected subconjunctivally below. Conjunctival sutures are finally inserted to fix the flap in position. 


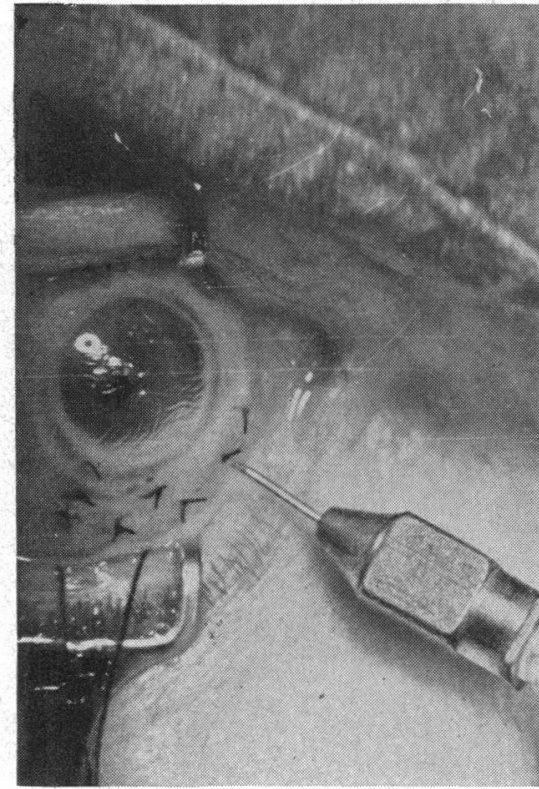

FIG. 4.-Hydrocortisone being injected into the anterior chamber, using a pipette made from a blood transfusion glass cannula, suitably cut and fitted with a rubber teat. The needle has been angled, the point cut off, and the end smoothed. It incidentally makes an excellent iris repositor.

Post-operative Care.-This includes Seclomycin twice daily for 5 days. I aim at keeping the pupil about $3 \mathrm{~mm}$. in diameter, and atropine is only used if the pupil is much smaller than this. After the bistamine-pilocarpine drops, the pupil is usually very small and ung. atropine usually produces a 3-mm. dilation after the first application. Should this not occur the atropine is continued twice or three times a day until the desired pupil size is obtained. If at the second dressing (first post-operative day) it is noted that the atropine has made no difference to the pupil size, and especially if there is exudate occluding the pupil, a more powerful mydriatic such as 1 per cent. adrenaline is required. (It must be preceded by a drop of local anaesthetic.) Rarely the pupil enlarges more than the $3 \mathrm{~mm}$. ideal and then a miotic is required.

Lens implant cases require more detailed care than straightforward cataract extractions, and twice-daily dressings are almost the rule for the first 4 to 5 days. My private cases as a group have better results than hospital cases where one had to rely on house surgeons to do the dressings. The hydrocortisone introduced into the anterior chamber lasts about 3 to 6 days depending upon the preparation. Topical ung. hydrocortisone is 
started if thought advisable at this time, provided all striate keratitis has cleared. With my first eight acrylic implants no intra-cameral cortisone was used and some fairly severe iritic reactions occurred, producing very dense occlusive pupillary membranes that required several needling operations to establish a clear pupil. Having previously experimented with injections of cortisone suspensions into the vitreous and anterior chamber without producing ill effects, I tried the procedure of introducing a few minims of the suspension $(25 \mathrm{mg} . / \mathrm{ml}$.) into the anterior chamber at the end of the operation. I found the results most pleasing, the iritic reaction being greatly reduced and the great majority of cases having a clear pupil. The usual acetate preparations of hydrocortisone take about 6 to 7 days to absorb completely. Occasionally, when a little more was introduced than usual, a denser clump formed on the iris which lasted longer. In two of these cases a small pucker developed in the iris where the hydrocortisone had remained clumped for 10 days.

The ordinary hydrocortisone acetate suspension contains various inert suspending agents such as carboxymethyl cellulose and 0.5 per cent. benzyl alcohol added as a preservative. Four of the failures to be described below developed a corneal dystrophy, and amongst other factors I suspected the benzyl alcohol. At my request Roussel Laboratories kindly made for me ampoules of hydrocortisone suspension without benzyl alcohol, using the free alcohol compound instead of hydrocortisone acetate. Some ampoules contained carboxymethyl cellulose as a suspending agent, and others contained the hydrocortisone compound in a very finely divided form in normal saline. It is the free alcohol form of hydrocortisone that reacts in the tissues and this preparation was therefore used to obtain the maximum antiphlogistic effect. This compound is absorbed from the anterior chamber in 2 to 4 days. The immediate result was better than with the hydrocortisone acetate preparation, but in a few cases a mild iritic reaction presented itself a few days after all the hydrocortisone had been absorbed. An ideal preparation would therefore probably be a mixture of the acetate and freealcohol compounds in equal parts. No case treated with this preparation developed corneal dystrophy but the number is too small to be sure on this point.

\section{Failures}

As mentioned above this series included twelve failures. In four the lens is in excellent position and the pupil is clear, but the cornea has undergone a degeneration identical with a Fuchs's dystrophy. Commencing as a severe striate keratitis, the lesion progressed to full-thickness corneal haze and eventually to epithelial haze and small bullae. Two of these four cases were operated upon the same morning and it was subsequently discovered that a solution prepared by adding salt tablets to boiled tap water had been used for the anterior chamber irrigation instead of the usual "Vacoliter" normal saline. The possibility of endothelial damage by a probably hypertonic solution cannot therefore be excluded. In the 
other two cases a fair amount of manipulation was necessary to centre the lens which persisted in sliding to one side or the other. A rather turgid vitreous bulged the posterior capsule forwards causing the lens to slide to the periphery. At that time centralization was achieved by applying pressure to the lens through the cornea, and this no doubt damaged the endothelium, while the hydrocortisone also probably delayed the healing of the damaged endothelium. It is for these reasons that I now get the eye as soft as possible pre-operatively and take every precaution to avoid damage to the endothelium. Additional topical cortisone therapy is not used until all striate keratitis has resolved. The anterior chamber irrigation must be kept to the minimum.

Two of these dystrophy cases have had corneal grafts. The one has achieved $6 / 12+$ vision. In the other the graft has hazed but the eye is comfortable and future grafting is contemplated. Of the other two cases, one has refused further treatment, and the other is using hypertonic local applications. The corneal haze in the latter is not very marked and the oedema is settling.

In five cases the lens luxated into the anterior chamber. These were all cases of traumatic cataract, four being aphakic when first seen. As news of successful implants spread, relatives and friends of such patients came to see if they could have their unilateral aphakia corrected. Some of them were really not suitableiris splits, broad posterior synechiae which were difficult to divide-and were warned that the lens might not stay in position and might have to be removed. In fact, the lenses had to be removed within 3 months from the four aphakic cases. Two unfortunately walked about for 2 to 3 weeks with the luxated lens resting against the endothelium. They too developed the Fuchs-like dystrophy mentioned above, though their corneae had healed uneventfully after the implant operation itself. The fifth luxation occurred 18 months after the original implant operation; in this case a traumatic cataract had been caused by an intra-ocular foreign body that had nicked the pupil margin at 12 o'clock. At the implant operation a rather broadish basal iridotomy-practically an iridodialysis - was done at 12 o'clock to allow the iris to sag a little to cover the upper pole of the lens, which was also centred well above the pupil centre. However, a puckering of the iris occurred round the upper pole of the acrylic implant, and despite the use of eserine drops the upper part of the iris was pulled up a little as a broadish posterior peripheral synechia formed between 10 and 2 o'clock. After 6 months the condition appeared static, with visual acuity $6 / 12$ and all seemed satisfactory, but a year later the patient reported that a week previously he had felt a sudden pain, and that the vision, which had improved considerably during the year, had now deteriorated. The upper pole of the lens was found resting against the endothelium. The lens had apparently dropped a little more and the contracting posterior synechia above had broadened the gap caused by the small nick in the pupil edge, allowing the iris to slip over the upper pole of the lens. After removal of the lens the cornea, which had been hazy, settled satisfactorily. This is interesting when compared with the two cases that developed a corneal dystrophy, in which the endothelial contact had been present for 2 to 3 weeks.

Peripheral posterior synechiae causing the iris to pucker round the edge of the acrylic lens were noted in many cases. This is one reason for keeping the pupil contracted to about $3 \mathrm{~mm}$.

In two other instances an implant was done after an intracapsular extraction. 
Having heard that this was being done successfully, I first tried it on a patient who had developed acute glaucoma as a complication of a hypermature cataract. By the time of the operation there was no perception of light. The operation went off so well and the technical result was so pleasing that the same operation was done a month later in another patient in a seeing eye, and here too the result appeared to be excellent. However, a few weeks later the lens in the first case was seen to be sliding downwards between the iris and hyaloid membrane and it was soon obvious it would have to be removed. In the second case the same thing developed after several weeks and that lens too was therefore removed.

The last of this series of failures occurred in a case of heterochromic cyclitis. A moderately severe hyphaema occurred and left a rather dense occlusio pupillae. This was needled twice and a clear pupil was developing when glaucoma supervened. This did not respond medically and the patient refused further surgery. No other case of glaucoma as a complication was observed in this series.

Of these twelve failures, therefore, six or seven could have been avoided and improved technique should eliminate the rest. The intracapsular extraction cases are self-evident. Traumatic aphakia requires careful investigation and assessment: the iris sphincter must be active and intact and posterior synechiae must be easily divisible so that the acrylic lens can be evenly covered. The operation should only be attempted in cases of heterochromic cyclitis if haemorrhage seems unlikely and ocular tension is normal.

\section{Minor Complications}

Apart from the twelve failures described above, no other serious complication developed in this series.

Three cases developed a late haziness of the posterior capsule-after 2 years in one case, and after 1 year in two cases; posterior needling restored the vision fully in all three. Posterior needlings were done in 24 of this series; in 21, including fourteen of the traumatic cataract cases, about 3 months after the implant was done. This early posterior capsular haze is due to immaturity of the cataract and hyphaema.

The late hazing seen in three cases might have been due to the ingrowth of cells from tags of anterior capsule sandwiched between the lens and posterior capsule.

Needling of an anterior occlusio pupillae membrane was done in fifteen cases, six of which had had hyphaemas filling more than half the anterior chamber. Other large hyphaemas absorbed leaving no membrane, but some required several anterior or posterior needlings, and five had both anterior and posterior discissions.

The main point in the technique of posterior needling is focal spot illumination by means of a headlamp worn by the surgeon, which is adjusted before scrubbing up. A pre-operative miotic is advisable if the pupil is more than $3 \mathrm{~mm}$. in diameter. Retrobulbar anaesthesia is used, and the needle is inserted into the vitreous body about $5 \mathrm{~mm}$. posterior to the temporal limbus. The posterior capsule does not snap open when needled and a vertical as well as a horizontal cut is usually necessary to produce a small opening which is all that is necessary provided it is central. This requires careful pre-operative planning with the headlamp for illumination; a lamp held by an assistant is not satisfactory, the rays being too oblique. 
Most of my cases developed a greater or lesser degree of compound myopic astigmatism with the implant. The power of the Ridley lens is 73D in air which is much greater than that of the normal lens.

In many of my cases the lenses have dropped towards the 6 o'clock meridian of the posterior chamber. This has occurred despite an initial high positioning of the lens. Possibly the hydrocortisone, by inhibiting the formation of adhesions, favours the gradual sag of the acrylic downwards where it comes to rest on the ciliary body. This has not caused any reaction, but it means that the centre of the pupil in an average 12 to $13-\mathrm{mm}$. cornea is about $2 \mathrm{~mm}$. from the upper edge of the lens-diameter which measures $8.4 \mathrm{~mm}$. This is my main reason for keeping the pupil at the most about $3 \mathrm{~mm}$. in diameter. However, posterior peripheral synechiae have drawn the iris round the edge of the lens and in some cases the groove on the anterior surface of the acrylic is visible. Even in cases where the lens remains central, the relatively abrupt thick edge of the lens leaves a space between iris, posterior capsule, and lens edge. Retained lens matter, plus fibrin, can fill this space in parts, so favouring the formation of posterior synechiae, and puckering the iris. This in turn tends to displace the lens to one side or the other, distorting the pupil, and so favouring anterior dislocation.

\section{Suggestions for an Improved Lens}

For these reasons I consider a better form of lens to be of larger diameter $(9.4 \mathrm{~mm}$.) and thinner $(1.5$ instead of $2.4 \mathrm{~mm}$.). This allows a thin taper edge instead of the relatively thick abrupt edge of the present lens. A wider area of separation of iris and posterior capsule is thereby achieved and should a peripheral posterior synechia form, any iris pucker around the thinner edge of the lens can be only very slight. Centration of the lens is easier, so that little manipulation is required, and the risk of astigmatism resulting from oblique and peripheral centration is less. The thinner lens means less forward bulging of the iris, which may cause occlusion of the angle and the development of glaucoma.

A new series of cases is now being tried with such a lens; its other measurements are anterior curvature $20 \mathrm{~mm}$. and posterior curvature $12 \mathrm{~mm}$. The results so far seem pleasing but the number of cases is not yet sufficient to allow definite conclusions to be drawn.

\section{Summary}

The results in 84 Ridley lens implant operations are discussed. Twelve failures are analysed with suggestions how to avoid them. Six of these twelve cases were of an experimental nature. Aspects of technique are described, including the value of Diamox pre-operatively, and hydrocortisone into the anterior chamber. A modified lens is suggested to give better refractive results, facilitate centration, and eliminate the bad effects of posterior peripheral synechiae. 